\title{
LRP2 a myopic hotspot between environment and genetics
}

\begin{abstract}
Myopia is typically due to the excessive growth of the eye resulting in blurred vision of distant objects. High myopia (HM) and its associated pathologies, i.e glaucoma, retinal detachments or myopic retinopathy are important causes of vision loss especially in young adults and their incidence is constantly on the rise. The mechanisms that regulate myopic eye growth are poorly understood but inherited, environmental and behavioral factors may contribute to the development of HM. HM is a cardinal feature of the Donnai-Barrow syndrome (DBS), a rare autosomal recessive condition combining facial dysmorphology, sensorineural hearing loss and proteinuria. DBS is caused by mutations in LRP2 encoding the low density lipoprotein receptor-related protein 2 (LRP2), a large endocytic receptor strongly expressed in the ocular epithelia and involved in the handling of various molecules including retinal, transthyretin, leptin or vitamin D. The ocular manifestations of DBS patients or mice lacking LRP2 specifically in the eye include excessively enlarged globes, increased axial length, retinal degeneration and detachments and normal intraocular pressure. Impaired formation of the endocytic apparatus is a common feature in the renal epithelia of DBS patients and the retinal pigment epithelium of the LRP2-deficient eyes suggesting that impaired transport or clearance of LRP2 ligands in the ocular tissues may be involved in myopia induction and progression. In this review we summarize current knowledge on myopia development and propose that modulating endocytosis of LRP2 and its ligands in the ocular tissues may be considered to prevent or control HM and its associated complications.
\end{abstract}

Keywords: donnai-barrow syndrome, donnai barrow, endocytosis, high myopia, LRP2, low-density lipoprotein receptor-related protein 2, megalin, myopia, RPE, transcytosis, vitreous
Volume 7 Issue I - 2017

\author{
Renata Kozyraki, Olivier Cases \\ Paris-Diderot University, France
}

Correspondence: Renata Kozyraki, Inserm Umrs_I 138, 15 rue de I,Tel + 33 I44278007, Email renata.kozyraki@inserm.fr

Received: May 19,2017 | Published: June 08, 2017
Abbreviations: $\mathrm{AL}$, axial length; DBP, vitamin D binding protein; DBS, donnai-barrow syndrome; HM, high myopia; IGF, insulin-like growth factor; LRPAP-1, low-density lipoprotein receptorRelated protein associated protein 1; LRP2, low-density lipoprotein receptor-related protein 2; NPE, non-pigmented epithelium, PE, pigmented epithelium; RAP, receptor-associated protein; RBP, retinol binding protein; RPE, retinal pigment epithelium; TGF, transforming growth factor; VEGF, vascular endothelial growth factor

\section{Introduction}

Myopia is a complex disease resulting from the interaction of multiple genetic and environmental factors. ${ }^{1-3}$ The incidence of myopia increased dramatically over the last decades; moreover the prevalence of HM (i.e. axial length superior to $26 \mathrm{~mm}$; refractive error of less than -6 diopters) is particularly elevated. Genetic studies associated growth factors such as Transforming Growth Factor (TGF-beta), Insulin-like Growth Factor (IGF) or Vascular Endothelial Growth Factor (VEGF) with myopia and HM. The increasing prevalence of the disease is thought however to be driven by environmental factors such as light levels, life style or diet. ${ }^{3,4}$ Light levels are inversely associated with myopia progression and this is mainly associated with the release of retinal dopamine, a potent regulator of eye growth. A minimal contribution of low systemic vitamin D levels is also described but further studies are required to support this hypothesis. An obesogenic diet and sedentary life style may influence both the development of metabolic disorders and the progression of myopia most likely by altering insulin resistance and the expression of genes that influence insulin sensitivity, such as adiponectin or leptin. Experimental studies indeed showed that intravitreal injections of insulin result in increased axial elongation of the eye, the principal feature of myopia.,

Myopia and HM affect both children and young adults. Myopic and especially highly myopic eyes are more susceptible to pathological ocular changes including cataract, glaucoma, retinal detachments and myopic macular degeneration, all of which can lead to legal blindness. $\mathrm{HM}$ is the fourth most common cause of irreversible vision loss and a public health problem imposing an enormous economic and social burden in many countries including western ones, China or Japan..$^{7-10}$ Despite active research the exact mechanisms that govern myopic eye growth are still unclear. Understanding how the genetic and environmental factors are continually adjusted to match eye size with refractive power will allow preventing, controlling and/or treating the development of HM and its complications.

\section{Discussion}

\section{Mechanism of myopia induction}

Disruption of the emmetropization process, i.e. the precise coordination of axial length (AL), corneal curvature, lens thickness 
and anterior chamber depth results to refractive errors such as myopia. Normal and myopic postnatal eye growth are generally thought to be vision-guided and controlled locally. ${ }^{8}$ Retina plays a central role in the development of myopia and the increase of AL (of the posterior segment particularly), the major determinant of the degree of myopia. Numerous experimental studies clearly showed that defocus of the peripheral retina selectively acts on the optical axis. Retinal perception of the defocus evokes signals that are transmitted to the choroid and sclera to favor the progression of myopia. ${ }^{11}$ Several elements of the processes involved are still missing but some others are supported by experimental and genetic studies. The amacrine cells and gene products of the WNT signaling pathway as well as retinal messengers including dopamine and retinoic acid are thought to be essential in the process by acting on different parts of the eye. ${ }^{8}$ In this scheme, the retinal pigment epithelium (RPE), critical for photoreceptor function and regulated ion and fluid exchange between the retina and the choroid, would relay the signals to the sclera. RPE cells express indeed receptors for dopamine, can metabolize retinol and other molecules essential in ocular tissue homeostasis including vitamin D. Additionally, RPE is a major source of the myopia-associated factors IGF-1, TGF-beta or VEGF. ${ }^{12-14}$ The polarized secretion of these factors toward the retina or the choroid depends on RPE integrity and is critical for retinal and choroidal health.

Other parts of the eye that may be involved in the induction of myopia are the ciliary body and its products the aqueous and vitreous humor. Modifying the transparency and/or biochemical composition of these optical media could perturb refractive growth Increased levels of DBP (vitamin D binding protein), albumin and transthyretin, established LRP2 ligands (see below) in the aqueous of highly myopic eyes are proposed as HM biomarkers. ${ }^{15}$ With respect to the vitreous, experimentally induced vitreous liquefaction leads to increased $\mathrm{AL}^{16}$ and naturally occurring extensive vitreous liquefaction in man is commonly associated with the evolution of HM and its associated pathologies. ${ }^{17}$ The cause of these modifications is not clear; nevertheless recent studies ${ }^{18,19}$ suggest that altered synthesis or local handling of aqueous and vitreous components may participate in the development of myopia.

\section{Donnai-Barrow syndrome}

Loss of function mutations in LRP2 lead to the Donnai-Barrow syndrome (DBS), a rare disorder characterized among others by facial dysmorphology, HM often leading to retinal detachment, sensorineural hearing loss, developmental delay and proteinuria. Congenital diaphragmatic hernia and/or omphalocele are present in $50 \%$ of the affected individuals ${ }^{20}$ Facial features include hypertelorism, mid face hypoplasia, prominent eyes, down slanting palpebral features, a short nose and posteriorly angulated ears. Ophthalmological examination reveals bilateral HM (up to -20 diopters), retinal dystrophy, and in some cases iris coloboma, vitreous opacities and cataract. Intraocular pressure is within the normal range. Progressive visual loss is common in DBS but prompt treatment can improve the chances of useful vision. Prophylactic treatment with peripheral barrier photocoagulation has successfully been used to prevent retinal detachment. Retinal dystrophy has also been observed but its nature is not clearly determined. Information on long-term follow up of DBS patients is indeed limited: many affected pregnancies are interrupted or result in perinatal death. To date 10 different LRP2 mutations associated with DBS are reported and either lead to aberrant LRP2 synthesis or abolish its ligand-binding capacities. Increased excretion of the known LRP2 ligands retinol-binding protein (RBP) and DBP are characteristic of DBS patients and essential for diagnosis. Current data suggest that increased excretion of these carriers may result in diminished blood levels of vitamins $\mathrm{A}$ and $\mathrm{D}$ in the patients.

\section{LRP2}

Low density lipoprotein (LDL) receptor-related protein 2 (LRP2) is an evolutionary conserved transmembrane protein of the LDL receptor gene family. ${ }^{21}$ Mammalian LRP2 is strongly expressed at the apical surface of various absorptive epithelia such as the renal, the ocular, RPE and non-pigmented ciliary epithelia, or the ependymal cells of the brain. ${ }^{18,21-24}$ Known LRP2 ligands are numerous plasma proteins, vitamins or steroid carriers including RBP, DBP or the signaling molecules Shh and BMP4. ${ }^{21}$ LRP2 ligands such as transthyretin, albumin, insulin, leptin or angiotensin II are implicated in HM, diabetes, obesity, hypertension and other pathological conditions which may affect both renal function and retinal health. With the exception of the renal effects of LRP2 dysfunction which have been extensively studied, the pathological mechanisms that relate LRP2 to other systems are not exactly defined. ${ }^{25-28}$

\section{LRP2-mediated endocytosis}

In the kidney, LRP2-mediated tubular reabsorption of plasma proteins filtered by the glomeruli is a prime step for vitamin and nutrient homeostasis. ${ }^{22}$ LRP2-ligand endocytosis is followed by lysosomal ligand degradation and recycling of the cargo-free receptor to the plasma membrane (Figure 1). This process is essential for vitamin D maturation and global vitamin homeostasis; this observation is further supported by the low blood levels of vitamin A and D in DBS patients..$^{20}$ In other sites such as the thyroid epithelium or the microvessel endothelial cells of the blood-brain barriers, the LRP2ligand complexes enter the transcytosis pathway. In these cases the internalized receptor reaches the baso-lateral plasma membrane and the ligand is released in the extracellular milieu without degradation (Figure 1).

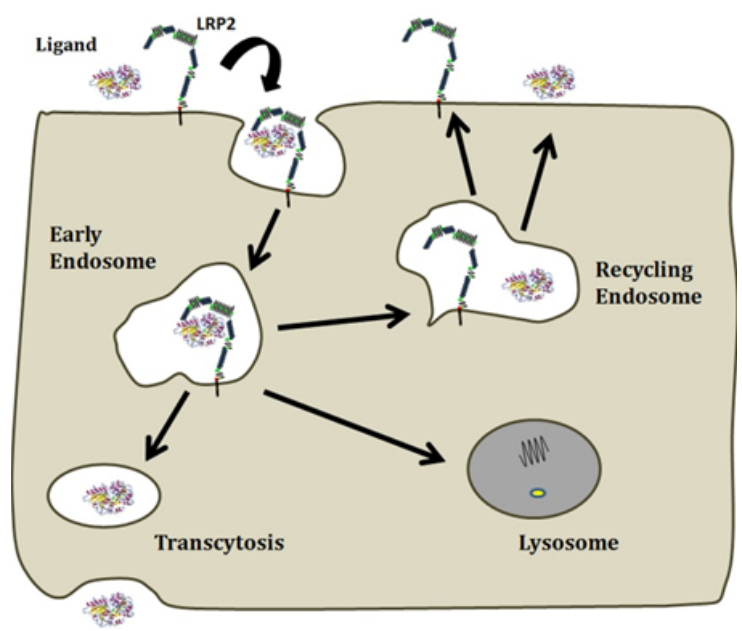

Figure I Following LRP2/ligand-mediated endocytosis via apical coated pits, the complexes accumulate in early endosomes whereby they are sorted out in different routes. First, the drop in luminal $\mathrm{pH}$ disrupts binding of ligands to LRP2, enabling receptors to recycle to the apical membrane while the ligand moves to lysosomes for degradation. However, some LRP2/ligand complexes resist to low $\mathrm{pH}$ and recycle causing resecretion of the ligand. $\mathrm{A}$ third route involves the complex trafficking to the baso-lateral membrane, resulting in the transcytosis of the ligand. 
Within the RPE, LRP2 is mainly expressed at the apical pole facing the subretinal space. Some signal is also seen in basolateral vesicles suggesting that LRP2 may be involved in both endocytosis and transcytosis events between the retina and the choroid. Supporting LRP2 implication in basolateral endocytosis intravenously injected fluorescent albumin rapidly accumulates in endosomal vesicles of the RPE. In the ciliary body LRP2 is strongly expressed at the interface between the non-pigmented (NPE) and pigmented ciliary epithelium (PE). Whereas the NPE cells are interconnected by tight junctions and form an impermeable cell layer, the PE cell layer is leaky allowing for the passage of macromolecules from the blood to the NPE. The function of LRP2 at this site has not been studied; nevertheless it is tempting to propose that LRP2-mediated degradation of various serum proteins contributes to the formation of the blood-aqueous barrier and the blood-retinal barrier as well as the selective transport of vitamins and other nutrients through the NPE and RPE cell layers.

\section{Animal models of LRP2 inactivation}

To uncover the function of LRP2 germ-line inactivated or mice carrying a floxed LRP2 allele were generated. ${ }^{18,29-31}$ Supporting a crucial role of LRP2 in nutrient uptake and vitamin and lipid homeostasis, the LRP2 null mice have a low survival rate, facial dysgenesis, defective forebrain development and impaired pulmonary and kidney function. ${ }^{29,32}$ Epiblast-specific inactivation of LRP2 via the MORE-cre results in a very similar phenotype clearly indicating the necessity of LRP2 in the embryonic tissues. ${ }^{30}$ Mice lacking LRP2 in the brain microvessel endothelial cells present with cortical neurodegeneration and exhibit mild obesity and elevated leptin and insulin concentration further underlying the contribution of LRP2 in both metabolic and neurodegenerative diseases in the adult. ${ }^{28,33}$

\section{LRP2 and high myopia}

Nonsense mutations in the zebrafish $\operatorname{lrp} 2$ gene lead to adult onset progressive myopia, increased intraocular pressure and defective renal protein reabsorption. Eye growth is most likely due to the elevated IOP and is accompanied by retinal thinning. Despite the phenotypic differences between the myopic eyes of DBS patients and the enlarged ocular globes of the zebrafish mutant this model was the first to suggest that $\operatorname{lrp} 2$ dysregulation is the cause of eye enlargement. ${ }^{34}$ More recently, targeted ablation of LRP2 from the ocular tissues via the FoxG1::Cre mouse line was reported. FoxG1::Lrp2 mice display aberrant eye growth associated with excessive and continuous elongation of the vitreal chamber, chorioretinal atrophy, scleral thinning and posterior staphyloma, features reminiscent of myopic retinopathy. In these mice the intraocular pressure is not modified but the number of photoreceptor, bipolar and retinal ganglion cells is severely affected resulting in progressive optic nerve atrophy. ${ }^{18}$

The onset of the LRP2-deficient eye growth is precocious, i.e. during the first week of post-natal life, before the eyes open and may be light- but not vision-guided. The rate of axial and vitreal chamber elongation is however particularly high during the week that follows eye-lid opening, i.e. between post-natal day 15 (P15) and P21, suggesting that impaired handling of ocular signals contributes to the progression of myopia in this mouse model. Although this observation may suggest that LRP2 is involved in the transmission and/or reception of locally generated signals, further experiments, using for instance negative-powered lenses or translucent diffusers, will be necessary to clarify this point. As previously stated LRP2 is expressed at the basolateral pole of the RPE cells where it may be involved in endocytosis of serum-derived macromolecules. In agreement with this hypothesis the uptake of intravenously injected fluorescent albumin was completely abolished in the LRP2-deficient RPE cells. ${ }^{19}$ Moreover the uptake of fluorescent transferrin was also impaired despite the expression of the cognate transferrin receptor in these cells. Finally, the formation of endosomal and lysosomal vesicles was severely compromised in the mutant RPE pointing to LRP2-mediated endocytosis as a key player in normal RPE exchanges with the choroid and systemic circulation. ${ }^{19}$

The exact function of LRP2 expressed at the apical pole of RPE cells remains unknown. However, transmission electron microscopy analysis revealed that the LRP2-deficient RPE cells lack long apical microvilli and that the outer rod segments are absent, suggesting pathological modifications of the subretinal space. Additionally the formation of the junctional complexes between RPE cells is compromised and the cells present signs of apical de-differentiation. ${ }^{19}$ These observations are consistent with the implication of LRP2 in RPE polarity and photoreceptor health. LRP2 may also be involved in the exchange/clearance of visual cycle components between the photoreceptors and the RPE and/or participate in the RPE-retina signaling.

Because the composition of the vitreous gel reflects the health or disease status of the surrounding tissues, including the RPE we analyzed the vitreal chamber content of the LRP2-deficient eyes. In both control and mutant mice a gel and a liquid fraction were identified. ${ }^{19}$ The biochemical synthesis of the vitreous gel fraction was similar in control and mutant eyes. In the myopic eyes however the amount of the liquid fraction was dramatically increased and its ionic and protein composition were impaired. Despite normal systemic values the abundance of potassium, sodium and chloride were significantly decreased whereas the concentration of phosphorus and calcium were increased. It is tempting to associate the above modifications with impaired fluid transport across the Müller and RPE cells of the LRP2-deficient eyes. Further experiments are required to evaluate the values of the same electrolytes within the retinal tissues and associate them with the progression of the disease.

The protein composition of the mutant liquid vitreous was also dramatically modified. In addition to the strong down-regulation of proteins involved in photoreceptor differentiation and function, proteins involved in myopia susceptibility such as the amyloid beta precursor-like protein 2 and retinaldehyde dehydrogenase 2 were almost completely undetectable in the mutant vitreous. ${ }^{19}$ It is of interest that several LRP2 ligands, including albumin, transthyretin or DBP, previously reported to be upregulated in the ocular fluids of patients with HM were also increased in the mutants. The increase of LRP2 ligands in the ocular fluids may result from increased local production (synthesis or transport from the blood) or impaired degradation due to LRP2 absence or dysfunction. In this context it is interesting to note that mutations in LRPAP1, encoding the LRP intracellular chaperone RAP, were recently shown to be associated with HM. ${ }^{35}$ RAP is absolutely required for biosynthesis and plasma membrane expression of LRP2; it may thus be assumed that the above mutations indirectly result in LRP2 dysfunction and impaired endocytosis by abolishing its membrane expression.

\section{Conclusion}

Clinical and experimental evidence clearly indicates that LRP2 and its partners form a network essential for the regulation of normal 
and myopic eye growth. Several elements are still missing: the function of LRP2 expressed in the retinal ganglion or Müller cells is still unclear; another caveat is whether retinal degeneration separately results from LRP2 deficiency or whether it is a consequence of the myopic eye growth. Some other elements emerge: in the RPE cells LRP2 seems to be indispensable for the establishment of the endocytic apparatus and the internalization of blood- and most likely retina-derived metabolites. The severe apical dedifferentiation of the LRP2-deficient RPE cells further suggests that LRP2 is involved in the apical-basal organization of this monolayer and is therefore required for its functions. Because of its multilig and properties LRP2 may influence various metabolic processes; enhanced LRP2 expression and increased catabolism or intracellular transport of its ligands could be protective in various diseases including chronic kidney disease, diabetes, obesity or hypertension. It is tempting to propose that pharmacological induction of LRP2 expression may also modulate eye growth directly or indirectly via the regulation of insulin or retinol-associated signaling pathways. Controlling the progression of myopia is challenging; the pathogenic mechanisms operating at the molecular level are however not well understood and there is no efficient universal intervention that can prevent, delay the development or treat this pathology. In this respect, LRP2 by linking genetic myopia to environmental conditions may allow to shed some light on the pathways that regulate myopic eye growth.

\section{Acknowledgments}

This study was supported by Institut National de la Santé et de la Recherche Médicale (INSERM) and Centre National de la Recherche Scientifique (CNRS).

\section{Conflicts of interest}

The authors declare no conflicts of interest.

\section{References}

1. Williams KM, Hysi PG, Yonova-Doing E, et al. Phenotypic and genotypic correlation between myopia and intelligence. Sci Rep. 2017;7:45977.

2. Morgan IG, He M, Rose KA. Epidemic of pathologic myopia: What Can Laboratory Studies and Epidemiology Tell Us? Retina Phila Pa. 2017;37(5):989-997.

3. Young TL. Molecular genetics of human myopia: an update. Optom Vis Sci. 2009;86(1):E8-E22.

4. Young TL, Metlapally R, Shay AE. Complex trait genetics of refractive error. Arch Ophthalmol. 2007;125(1):38-48.

5. Feldkaemper MP, Neacsu I, Schaeffel F. Insulin acts as a powerful stimulator of axial myopia in chicks. Invest Ophthalmol Vis Sci. 2009;50(1):13-23.

6. Zhu X, Wallman J. Opposite effects of glucagon and insulin on compensation for spectacle lenses in chicks. Invest Ophthalmol Vis Sci. 2009;50(1):24-36.

7. Hawthorne FA, Young TL. Genetic contributions to myopic refractive error: Insights from human studies and supporting evidence from animal models. Exp Eye Res. 2013;114:141-149.

8. Morgan IG, Ohno-Matsui K, Saw SM. Myopia. Lancet. 2012;379(9827):1739-1748.
9. Holden BA, Fricke TR, Wilson DA, et al. Global Prevalence of Myopia and High Myopia and Temporal Trends from 2000 through 2050. Ophthalmology. 2016;123(5):1036-1042.

10. Aller TA. Clinical management of progressive myopia. Eye (Lond). 2014;28(2):147-153.

11. Summers JA. The choroid as a sclera growth regulator. Exp Eye Res. 2013;114:120-127.

12. Rymer J, Wildsoet CF. The role of the retinal pigment epithelium in eye growth regulation and myopia: a review. Vis Neurosci. 2005;22(3):251261.

13. Zhang $\mathrm{K}$, Zhu X, Chen M, et al. Elevated transforming growth factor- $\beta 2$ in the aqueous humor: a possible explanation for high rate of capsular contraction syndrome in high myopia. J Ophthalmol. 2016:5438676.

14. Zhu Y, Zhang T, Xu G, Peng L. Anti-vascular endothelial growth factor for choroidal neovascularisation in people with pathological myopia. Cochrane Database Syst Rev. 2016;12:CD011160.

15. Duan X, Lu Q, Xue P, Zhang H, et al. Proteomic analysis of aqueous humor from patients with myopia. Mol Vis. 2008;14:370-377.

16. Halfter W, Winzen U, Bishop PN, et al. Regulation of eye size by the retinal basement membrane and vitreous body. Invest Ophthalmol Vis Sci. 2006;47(8):3586-3594.

17. Holekamp NM. The vitreous gel: more than meets the eye. Am $J$ Ophthalmol. 2010;149(1):32-36.

18. Cases O, Joseph A, Obry A, et al. Foxg1-Cre Mediated Lrp2 Inactivation in the developing mouse neural retina, ciliary and retinal pigment epithelia models congenital high myopia. PloS One. 2015;10(6):e0129518.

19. Cases O, Obry A, Ben-Yacoub S, et al. Impaired vitreous composition and retinal pigment epithelium function in the FoxG1::LRP2 myopic mice. Biochim Biophys Acta. 2017;1863(6):1242-1254.

20. Kantarci S, Al-Gazali L, Hill RS, et al. Mutations in LRP2, which encodes the multiligand receptor megalin, cause Donnai-Barrow and facio-oculoacoustico-renal syndromes. Nat Genet. 2007;39(8):957-959.

21. Christ A, Herzog K, Willnow TE. LRP2, an auxiliary receptor that controls sonic hedgehog signaling in development and disease. Dev Dyn Off Publ Am Assoc Anat. 2016;245(5):569-579.

22. Kozyraki R, Cases O. Vitamin B12 absorption: mammalian physiology and acquired and inherited disorders. Biochimie. 2013; 95(5):1002-1007.

23. Lundgren S, Carling T, Hjälm G, et al. Tissue distribution of human gp330/ megalin, a putative $\mathrm{Ca}(2+)$-sensing protein. $J$ Histochem Cytochem. 1997;45(3):383-392.

24. Assémat E, Châtelet F, Chandellier J, et al. Overlapping expression patterns of the multiligand endocytic receptors cubilin and megalin in the CNS, sensory organs and developing epithelia of the rodent embryo. Gene Expr Patterns GEP. 2005;6(1):69-78.

25. Hama H, Saito A, Takeda T, et al. Evidence indicating that renal tubular metabolism of leptin is mediated by megalin but not by the leptin receptors. Endocrinology. 2004;145(8):3935-3940.

26. Shao J, Xin Y, Yao Y, et al. Functional analysis of misfolded transthyretin extracted from abnormal vitreous with high myopia related ocular pathologies. Clin Chim Acta. 2013;415:20-24.

27. Byun K, Gil SY, Namkoong C, et al. Clusterin/ApoJ enhances central leptin signaling through Lrp2-mediated endocytosis. EMBO Rep. 2014;15(7):801-808. 
28. Bartolome F, Antequera D, Tavares E, et al. Obesity and neuroinflammatory phenotype in mice lacking endothelial megalin. $J$ Neuroinflammation. 2017;14(1):26.

29. Willnow TE, Hilpert J, Armstrong SA, et al. Defective forebrain development in mice lacking gp330/megalin. Proc Natl Acad Sci U S A. 1996;93(16):8460-8464.

30. Spoelgen R, Hammes A, Anzenberger U, et al. LRP $2 /$ megalin is required for patterning of the ventral telencephalon. Dev Camb Engl. 2015;132(2):405-414.

31. Gajera CR, Emich H, Lioubinski O, et al. LRP2 in ependymal cells regulates BMP signaling in the adult neurogenic niche. $J$ Cell Sci. 2010;123(Pt 11):1922-1930.
32. Nykjaer A, Dragun D, Walther D, et al. An endocytic pathway essential for renal uptake and activation of the steroid 25-(OH) vitamin D3. Cell. 1999;96(4):507-515.

33. Dietrich M, Antequera D, Pascual C, et al. Alzheimer's disease-like impaired cognition in endothelial-specific megalin-null mice. J Alzheimers Dis. 2014;39(4):711-717.

34. Veth KN, Willer JR, Collery RF, et al. Mutations in zebrafish lrp2 result in adult-onset ocular pathogenesis that models myopia and other risk factors for glaucoma. PLoS Genet. 2011;7(2):e1001310.

35. Aldahmesh MA, Khan AO, Alkuraya H, et al. Mutations in LRPAP1 are associated with severe myopia in humans. Am J Hum Genet. 2013;93(2):313-320. 